\title{
Three-Dimensional Echo-Planar Spectroscopic Imaging
}

National Cancer Institute

\section{Source}

National Cancer Institute. Three-Dimensional Echo-Planar Spectroscopic Imaging. NCI

Thesaurus. Code C127837.

A three-dimensional application of magnetic resonance spectroscopic imaging that uses

an alternating gradient applied along one of the spatial readout directions to

simultaneously encode the space and chemical shift, thereby reducing the phase-

encoding dimension by one and increasing speed. 